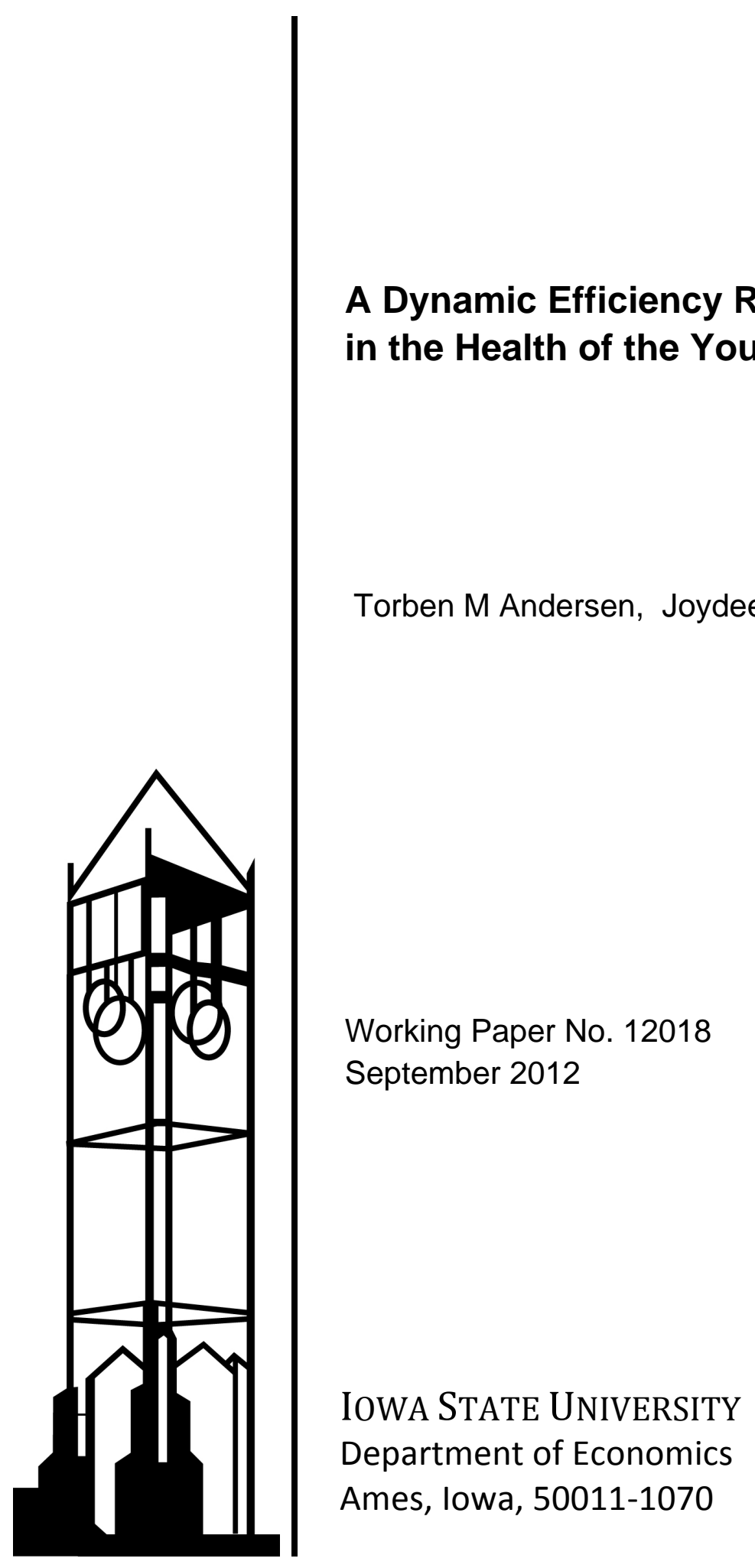

lowa State University does not discriminate on the basis of race, color, age, religion, national origin, sexual orientation, gender identity, genetic information, sex, marital status, disability, or status as a U.S. veteran. Inquiries can be directed to the Director of Equal Opportunity and Compliance, 3280 Beardshear Hall, (515) 294-7612. 


\title{
A Dynamic Efficiency Rationale for Public Investment in the Health of the Young
}

\author{
TORBEn M. ANDERSEN* \\ University of Aarhus, Denmark \\ CEPR, CESIFO, AND IZA \\ JOYDEEP BHATTACHARYA ${ }^{\dagger}$ \\ IOWA State University, USA \\ Revised version: September 24, 2012
}

\begin{abstract}
In this paper, we assume away standard distributional and static-efficiency arguments for public health, and instead, seek a dynamic efficiency rationale. We study a lifecycle model wherein young agents make health investments to reduce mortality risk. We identify a welfare rationale for public health under dynamic efficiency and exogenous mortality even when private and public investments are perfect substitutes. If health investment reduces mortality risk but individuals do not internalize its effect on the life-annuity interest rate, the "Philipson-Becker effect" emerges; when the young are net borrowers, it works together with dynamic efficiency to support a role for public health.
\end{abstract}

${ }^{*}$ Corresponding author: Torben M. Andersen, Department of Economics, Building 322, Unversity of Aarhus, 8000 Aarhus C, Denmark; Phone: +45 8942 1609; Fax: +45 8613 6334; E-mail: tandersen@econ.au.dk

${ }^{\dagger}$ Joydeep Bhattacharya, Department of Economics, Iowa State University, Ames IA 50011-1070, USA. Phone: (515) 294 5886, Fax: (515) 294 0221; E-mail: joydeep@iastate.edu 


\section{INTRODUCTION}

In this paper, we explore a dynamic-efficiency rationale for public health spending on the young. Health experts and economists have identified two economic rationales - broadly classified as enhancing "static efficiency" - for public involvement in health. First, some actions that promote health - vaccinations, smoking-cessation programs, and so on - are public goods or generate considerable positive externalities, and hence, would be underprovided by private markets. Second, market failures of all sorts, both in the provision of care and in health insurance, may justify governmental intervention. ${ }^{1}$ Add to that list, equity concerns: health affects productivity and well-being, and the poor, unable to afford much health care, may remain stuck in poverty. Health care is a basic service essential in any effort to combat poverty, and as such, public funds may be needed to help achieve that aim. This paper addresses the following issue: setting aside the "static efficiency" rationales and distributional concerns (and assuming perfect capital markets), can dynamic efficiency considerations justify public investment in health?

The presence of the government in matters relating to overall health is huge. The United States government currently spends roughly $7 \%$ of its G.D.P on health care. The W.H.O estimates, on average, governments spend $4.6 \%$ of their country's G.D.P on health, the figure being as low as $1 \%$ in South Asia and as high as $8 \%$ in Western Europe. The World Development Report, Investing in Health (1993), documents that nearly $60 \%$ of world spending on health is disbursed via public health programs. Ignoring medical research, public involvement in health involves three sorts of investments, those in preventive, palliative, and curative care. Preventive medicine or preventive care refers to measures taken to prevent illnesses (and thereby, promote longevity and general good health) rather than curing them (curative care) or treating their symptoms (palliative care). Generally speaking, the relative importance of one kind of care over others, changes over the life cycle. Preventive health interventions typically happen early on in a life-cycle and are mostly intended to reduce subsequent morbidity and mortality risk. ${ }^{2}$ In the United States, nearly half of the nation's spending for personal health care goes to people aged 44 and below. It is impossible

\footnotetext{
${ }^{1}$ To this list, it is possible to add one more rationale - complementarities between private and public efforts to improve health; if such complementarity is strong (say, smokers are more likely to quit if their own effort is supplemented by government-run, smoking-cessation programs), it is again relatively easy to justify governmental intrusion in health.

${ }^{2}$ Preventive interventions are not restricted to vaccinations for infants and children; many affect the lives of adults late into life. Clinical preventive services include screening for high risk conditions, such as elevated blood lead levels, elevated blood pressure, and elevated serum cholesterol; management of certain communicable diseases, especially sexually transmitted diseases and tuberculosis; and counseling to reduce risky behaviors. Preventable causes of death, such as tobacco smoking, poor diet and physical inactivity, and alcohol abuse is responsible for nearly $40 \%$ of total yearly mortality in the United States.

Publicly-funded preventive health investments, such as counseling adults to quit smoking, screening for colorectal cancer, and providing influenza and other vaccinations, have been shown to have reduced mortality at very low cost. Public smoking-prevention efforts in the United States over the past three decades have been estimated to have saved 33 million person-years of life. See Gordon et. al (1996) for details.
} 
to point to an exact figure for the share of public resources directed to preventive health care or, more generally, to publicly-funded health care directed toward the younger generations.

The World Development Report (1993) argues that public action has contributed immensely to producing remarkable improvements in life expectancy, sharp reductions in child and adult mortality, and considerable increases in the disability-adjusted life years for many around the world. And yet, even as it endorses public health intervention, the report goes on to point out that "what people do with their lives [...] affects their health far more than anything governments can do". Given the sheer size of governmental involvement in health and the cautionary wrinkle introduced in the previous line, a broad question arises, should a government invest in health even when private agents are up to the task?

We pose versions of this question within the confines of a simple, two-period overlapping generations model of endogenous mortality and morbidity developed in the spirit of Grossman (1972), Ehrlich and Chuma (1990) and Philipson and Becker (1998). ${ }^{3}$ In the model economy, young agents invest a portion of labor income in their own health. Such preventive investments serve to reduce subsequent mortality risk and old-age morbidity; they also improve general well-being, ability to work, even earnings potential, later in life. ${ }^{4}$ We study the rationale of introducing a public-health program that tops up private health investment by the young and is financed by a distortionary tax on old (and young) labor income under a pay-as-you-go scheme. Perfect substitutability of private and public investment in health is assumed, and all the aforementioned static-efficiency justifications for public health are assumed away. In addition, perfect capital markets, including competitive, mortality-contingent claims ("life-annuity") markets, are assumed to exist.

To build intuition, first consider a benchmark setting with exogenous mortality risk. In this stripped-down economy, and under complete generality, we prove that introducing publicly-funded preventive health spending on the young is welfare-improving if the economy is initially dynamically efficient. (For our purposes, dynamic efficiency refers to a steady-state situation in which the gross real interest rate in an economy - the private opportunity cost of funds over time - is higher than the population growth rate, the corresponding social opportunity cost.) The result is derived for a reverse pay-as-you-go scheme that relies on past beneficiaries being taxed so that an intergenerational transfer mechanism (going from the old to the young) is active. The underlying intuition is that while the benefit of the public investment accrues to the young - among other things, it allows them to reduce their own private investments in health - its cost is borne by the

\footnotetext{
${ }^{3}$ For analyses of infectious dieases and health activites in OG models in the presence of external effects and capital market imperfections respectively, see e.g. Momota et. al (2005) and Augier and Yaly (2011).

${ }^{4}$ Private investment in one's health stock may include annual diagnostic health screening, opportunity cost of regular exercise, taking vitamins and nutrients, eating nutritious food, health benefits from quitting unhealthy habits, such as, smoking, and so on.
} 
old, partially or wholly. Since these taxes represent intergenerational transfers from the old to the young, the private opportunity cost of investment by the young is the interest rate, while the social opportunity cost of public investment is one (if the old pay for it entirely, and there is no population growth). In a dynamically-efficient economy, the former exceeds the latter, making the public option attractive at the margin. ${ }^{5}$

Next, we study a setting in which mortality risk is endogenous; in particular, young-age health investments reduce subsequent mortality risk. In Philipson and Becker (1988), the availability of mortality-contingent claims increases the incentive of agents to invest in their health, which in turn, increases their longevity. As originally noted by Davies and Kuhn (1992), moral hazard arises when such longevity-inducing behavior is undertaken without internalizing its effect on the premium paid by others. It follows, from society's standpoint, when mortality-contingent claims are present, private agents overinvest in their health. We call this the "Philipson-Becker effect". Evidently, when this effect is present, simple dynamic efficiency is no longer enough to make the case for public health. Indeed, the discussion gets more nuanced because the net asset position of agents starts to matter. To see this, consider a setting in which an increase in public health spending raises overall health spending even after the crowding out of private spending is accounted for. Such action reduces overall mortality risk, and hence, the interest rate on life annuities. This helps the young, if they are net borrowers, by easing their interest burden from past borrowing. It follows, from a societal perspective, the Philipson-Becker effect implies young net-borrowers underinvest in their own health. And public health can correct for this private underinvestment. ${ }^{6}$ In this case, the Philipson-Becker effect works in tandem with dynamic efficiency to support a role for public health. It bears emphasis that the importance of the net asset position of agents has not been highlighted, heretofore, in the literature.

Our paper fits well in a short line of research - starting with Davies and Kuhn (1992) and continued in Philipson and Becker (1998) and Liu, Rettenmaier, and Saving (2005) - on the moral hazard issue in annuity markets. Philipson and Becker (1998) analyze a model similar in many respects to ours, but unlike us, they study a pay-as-you-go (PAYG) pension (one that taxes the young and transfers a lump-sum to each of the surviving old). The fact that payment of the pension benefit is contingent on survival converts the pension into something like an annuity - in fact, they

\footnotetext{
${ }^{5}$ This result mirrors the classic Aaron-Samuelson result (Aaron, 1966) in pension economics. In that context, introducing a pay-as-you-go pension (funded by a lump-sum tax on the young) is welfare reducing if the economy is dynamically efficient. The upshot of the Aaron-Samuelson result is that in a dynamically-efficient economy, it is a bad idea to save via the pension system - the intergenerational transfer mechanism, going from the young to the old, hurts welfare. Our value added is to recognize that since saving via this transfer mechanism is bad, borrowing via the same scheme has to be good.

${ }^{6}$ In the model of Schneider and Winkler (2010), agents under invest in healthcare, as they fail to take into account that increased longevity in the aggregate induces a positive effect on the economy's growth rate.
} 
term social security as a "mandatory annuity program". Most importantly, though, the survival probability in their setup depends only on private health investments and not on anything that is publicly financed. They find that a more generous pension distorts private investments in health; it induces people to want to live longer. This causes private saving to go up, a result that goes against the standard intuition that pensions crowd out private saving and are welfare-reducing if the economy is dynamically efficient. Interestingly, Philipson and Becker (1998) consider a world with zero net interest and zero population rates of growth; in effect, their focus is restricted to the golden rule.

An important contribution is Zhang, Zhang, and Leung (2006) who extend Philipson and Becker (1998) by including production and allowing for two kinds of health investments, the kind the young make to reduce mortality risk and those made by the middle-aged to lengthen their working life. However, their focus, like that in Philipson and Becker (1998), is entirely on the general-equilibrium effects of a PAYG pension. Johannson (2000) studies insurance against health needs when old (curative and palliative) and not on preventive health investments activities as young - indeed, health status as old is not affected in any way by decisions made as young. Assuming perfectly inelastic labor supply and no private health insurance, he argues, based on steady-state welfare, the need for introducing PAYG health care, provided the old contribute to its financing. He goes on to compare a marginal introduction of private versus public health insurance (starting from an initial situation without any form of insurance) near the Golden rule and finds in favor of the private. We view Johannson (2000) as being complementary to the current paper.

The plan for the rest of the paper is as follows. In Section 2, the model setup is presented and an equilibrium is defined. Section 3 contains our main results concerning the optimality of public health investments, both for the case of exogenous and endogenous mortality. Section 4 concludes. Proofs of all the main results are contained in the appendix.

\section{THE MODEL}

2.1. Primitives. Consider a small open economy populated by a continuum of (potentially) two-period lived agents who are identical at birth. To keep the structure manageable, we merge the full life-cycle for individuals into two periods, denoted young and old. The young-age period may be thought of as youth and early adulthood combined, and the old, as mature adult and retired combined. Each agent is endowed with one unit of time in each period of life, i.e., both when young and when old. There is a single consumption good whose price is normalized to one. Agents are assumed to care about consumption and leisure in each period of life; in addition, they derive utility from their level of health when old. An agent survives onto the second period with probability $\pi$. 
When young, an agent can undertake preventive health investments designed to influence $\pi .^{7}$

The expected lifetime utility of an agent is given by

$$
u\left(c_{y}, 1-l_{y}\right)+\pi v\left(c_{o}, 1-l_{o}, h_{o}\right)
$$

where the subscript $y$ denotes "young" and $o$ denotes "old" and $u(\cdot)$ is a standard, concave felicity function defined on consumption when young $\left(c_{y}\right)$ and leisure $\left(1-l_{y}\right)$, where $l_{y}$ is labour supply. Likewise, $v$ is a standard, concave felicity function specified over consumption when old $\left(c_{o}\right)$, leisure $\left(1-l_{o}\right)$, and stock of health when old $\left(h_{o}\right)$. Note that utility as old under non-survival has been normalized to zero and $v>0$. Also, additive separability of utility is a convenient simplification; the thrust of our results is immune to the dropping of that assumption. Similarly, adding in an exogenously-specified discount rate in (1) has no qualitative consequences.

The assumption that old-age health status $\left(h_{o}\right)$ influences felicity incorporates different effects of morbidity on well-being: the marginal utility of consumption $\left(v_{c_{o}, h_{o}} \lesseqgtr 0\right)$ and of leisure $\left(v_{1-l_{o}}, h_{o} \lesseqgtr 0\right)$ may depend on health. The former captures the idea that pre-requisites for the enjoyment of consumption may depend positively or negatively on one's health. The latter, $\left(v_{1-l_{o}}, h_{o} \lesseqgtr 0\right)$, allows for the possibilities that a) better (worse) health may increase (decrease) the utility from time-consuming leisure activities (such as, travel) and b) decrease (increase) the disutility from work effort by making it easier (harder) to overcome the physical and physiological demands of the job.

An agent's health status when old is assumed to depend on health-specific investments made when young. ${ }^{8}$ These can be private - expenditure on items over which one has direct control, such as exercise, diet, vaccinations, preventive care, regular check-ups, protection against sexuallytransmitted diseases, smoking cessation, and the like. Or these can be public, incorporating almost any public health expenditure that directly affects private health, such as expenditure on vaccinations, smoking-cessation programs, the building of public hospitals that provide pre- and post operative care, and so on. We remain agnostic as to the precise nature of such investments. All we require is that the investments be made in the first period of life, and private agents, when making their own decisions, take the public investment as given.

We measure health expenditures in terms of consumption possibilities foregone for the sake of investment in health. Denote by $e_{y}^{p}$ the amount of private health investment, and by $e_{y}^{g}$, the level of

\footnotetext{
${ }^{7}$ In general, with heterogeneous individuals, the average mortality risk must be distinguished from an individual's mortality risk. Here, because all young are identical, in equilibrium, they each make the same level of investment, and hence, the economy-wide mortality risk $(\pi)$ coincides with that of any individual.

${ }^{8}$ Health investments by the old are disregarded so as to focus mainly on mortality-reducing preventive investments. Moreover, public health investments on the old are akin to public pensions which have been extensively studied in the literature.
} 
public investment. Private and public investments in health are assumed to be perfect substitutes; hence, total health investment is given by $e_{y} \equiv e_{y}^{p}+e_{y}^{g}$. The assumption of perfect substitutability between private and public investments in health is made so as to not bias the results in favor of public health activities. ${ }^{9}$ As will be evident, in equilibrium $e_{y}^{p}$ will respond to $e_{y}^{g}$ and may even get crowded out; in that event, a matter of some concern will be whether $e_{y}$ goes up or down.

Health investments made when young are assumed to affect both the survival probability (mortality) and the stock of old-age health capital (morbidity). The survival probability is assumed to depend on health investment, i.e., $\pi \equiv \pi\left(e_{y}\right)$ where $0<\pi \leq 1, \frac{\partial \pi}{\partial e_{y}} \geq 0$, and $\frac{\partial^{2} \pi}{\partial e_{y}^{2}} \leq 0$ holds. Similarly, we have that old-age health stock is given by $h_{o} \equiv h_{o}\left(e_{y}\right)$ where $\frac{\partial h_{o}}{\partial e_{y}} \geq 0$ and $\frac{\partial^{2} h_{o}}{\partial e_{y}^{2}} \leq 0$ holds. Increased morbidity is equivalent to a decline in $h_{o}$.

Agents are assumed to earn an exogenously-specified wage of $w_{y}$ in the first period of life. We allow for the possibility that earnings potential depends on health status so that the wage of the old is given by $w_{o} \equiv w_{o}\left(h_{o}\right)$ where $w_{o} \leq w_{y}, \frac{\partial w_{o}}{\partial e_{y}} \geq 0$, and $\frac{\partial^{2} w_{o}}{\partial e_{y}^{2}}<0$.

Denote aggregate saving by $S_{y}$, and by $R$, the gross, riskless return on funds between any two periods. We assume perfect capital mobility, and hence, $R$ is exogenously determined. Since agents face survival risk, being risk averse, they seek insurance. We posit all agents have access to, and exclusively use, both for borrowing and for saving, zero-cost intermediaries who operate in competitive, perfect annuity markets (as in Yaari, 1965); then the return to saving (or interest on borrowing) is $\tilde{R}$ where $\tilde{R}=R / \pi \cdot{ }^{10,11}$ Evidently, it will be important to note that private agents do not internalize the effect of $e_{y}$ on $\pi$, something the government does. For future reference, $R>1$ corresponds to dynamic efficiency, $R=1$ refers to the Golden rule, and $R<1$ implies dynamic inefficiency; see Blanchard and Fischer (1989).

The government funds public health investments, but no pension, via a distortionary tax $\tau$ $(0 \leq \tau<1)$ on young and old income. We assume the government operates under a balancedbudget requirement on a period-by-period basis, and is not permitted to borrow on the international

\footnotetext{
${ }^{9}$ There are plenty of examples of perfectly substitutable health activities; consider equally-good private and public hospitals coexisting, or governments handing out free smoking-cessation tools to smokers or condoms to those who visit sex workers, when those items may be bought at a private cost on the market, and so on. Perfect substitutability is, of course, an extreme assumption; it only makes it harder to rationalize the public component, the subject matter of this paper.

${ }^{10} \mathrm{On}$ the borrowing side, we assume private agents rely on funds from risk-neutral, competitive banks that borrow on the international market at the rate $R$ and, given the mortality risk, lend domestically to young borrowers at rate $\tilde{R}$, so as to break even. We (implicitly) assume that surviving borrowers cannot renege on paying back their debt.

${ }^{11}$ On the matter of exclusive use of annuities for transferring income across time, Davidoff, Brown and Diamond (2005) argue that rational risk-averse agents with access to both annuities and bonds, should save mostly via annuities. They also consider cases where a sudden medical need may arise at some point - a standard annuity does not provide insurance against such an event. If the contract does not provide insurance against such events, it may be optimal to save partly in bonds. In our case, health investment is a preventive activity and no such "health events" occur.
} 
market. The government budget constraint reads

$$
e_{y}^{g}=\tau w_{y} l_{y}+\tau \pi(\cdot) w_{o}\left(h_{o}\right) l_{o}
$$

For simplicity (and for realism), we assume the income tax rate is age-independent. Also note no claim is being made that such a tax is part of an optimal tax structure. To foreshadow, it will be important that the old contribute to the tax pool, i.e., some of the revenue needed to pay for public health investments on the young is raised from the old.

2.2. Agent's problem. The agent takes the tax rate $(\tau)$, the public level of health investment $\left(e_{y}^{g}\right)$, and the rate of return on annuities $(\tilde{R})$ as given. (In contrast, as discussed below, the policymaker will recognize the connection between $\tau$ and (2); in addition, she will internalize the effect of her choices on $\pi(\cdot)$ and hence on $\tilde{R}$.) The per-period budget constraints facing an agent are as follows:

$$
\begin{aligned}
& c_{y}=[1-\tau] w_{y} l_{y}-S_{y}-e_{y}^{p}, \\
& c_{o}=[1-\tau] w_{o} l_{o}+\tilde{R} S_{y} .
\end{aligned}
$$

The agent's problem is to choose $e_{y}^{p}, l_{y}, l_{o}$, and $S_{y}$ by maximizing (1) subject to these budget constraints, and the usual non-negativity constraints. $S_{y}$ is allowed to be positive (saving) or negative (borrowing).

The only decision facing the surviving-old is how much labor to supply $\left(l_{o}\right)$. Define

$$
V\left(e_{y}, S_{y}, \tau, \tilde{R}\right) \equiv \max _{l_{o}} v\left([1-\tau] w_{o}\left(h_{o}\right) l_{o}+\tilde{R} S_{y}, 1-l_{o}, h_{o}\left(e_{y}\right)\right)
$$

Noting $S_{y}$ is pre-determined, the first order condition to this problem (at an interior) reads

$$
v_{c_{o}}(\cdot)\left[[1-\tau] w_{o}\left(h_{o}\right)\right]-v_{1-l_{o}}(\cdot)=0
$$

and the second order condition is given by

$$
v_{c_{o} c_{o}}(\cdot)\left[[1-\tau] w_{o}\left(h_{o}\right)\right]+v_{\left(1-l_{o}\right)\left(1-l_{o}\right)}(\cdot)<0
$$

The salient properties of the indirect utility function $V$ are noted below:

$$
\begin{aligned}
V_{e_{y}} & =v_{h_{o}}(\cdot) \frac{\partial h_{o}}{\partial e_{y}}+v_{c_{o}}(\cdot)[1-\tau]\left[\frac{\partial w_{o}}{\partial h_{o}} l_{o}+w_{o}\left(h_{o}\right) \frac{\partial l_{o}}{\partial h_{o}}\right] \frac{\partial h_{o}}{\partial e_{y}}-v_{1-l_{o}}(\cdot) \frac{\partial l_{o}}{\partial h_{o}} \frac{\partial h_{o}}{\partial e_{y}} \\
& =\left\{v_{h_{o}}(\cdot)+v_{c_{o}}(\cdot)[1-\tau] \frac{\partial w_{o}}{\partial h_{o}} l_{o}\right\} \frac{\partial h_{o}}{\partial e_{y}}>0 \\
V_{S_{y}} & =\tilde{R} v_{c_{o}}(\cdot)>0 \\
V_{\tau} & =-v_{c_{o}}(\cdot) w_{o}\left(h_{o}\right) l_{o}<0 \\
V_{\tilde{R}} & =v_{c_{o}}(\cdot) S_{y} \lesseqgtr 0
\end{aligned}
$$


where (3) has been used. For future use, note from the expression for $V_{\tilde{R}}$ that the size and sign of $S_{y}$ matters, as is to be expected. This will be important because changes in public health investment will affect $\tilde{R}$, and hence utility, and the effect will depend on the size and sign of $S_{y}$. In particular, if $S_{y}$ is negative (the young are net borrowers), then $V_{\tilde{R}}<0$ holds; in this case, a rise in $\pi$ (subsequent to an increase in $e_{y}$ ) reduces $\tilde{R}$ and this helps the borrower (since the interest due on her past borrowing is now lower).

The young decide on labor supply, savings and health investments by maximizing expected life time utility:

$$
\max _{l_{y}, S_{y}, e_{y}^{p}} u\left([1-\tau] w_{y} l_{y}-S_{y}-e_{y}^{p}, 1-l_{y}\right)+\pi\left(e_{y}\right) V\left(e_{y}, S_{y}, \tau, \tilde{R}\right)
$$

subject to the aforementioned budget constraints, taking the tax rate $(\tau)$, the level of public health investment $\left(e_{y}^{g}\right)$, and the rate of return $(\tilde{R})$ as given. Even though $V(\cdot)$ is assumed to be concave in its arguments, it is by no means obvious that $\pi\left(e_{y}\right) \cdot V\left(e_{y}, S_{y}, \tau, \tilde{R}\right)$ is concave too.

Assumption $1 \pi\left(e_{y}\right) \cdot V\left(e_{y}, S_{y}, \tau, \tilde{R}\right)$ is strictly concave in $e_{y}$ and $S_{y}$.

In that case, the first order conditions for this problem (interior) are given by:

$$
\begin{aligned}
l_{y} \quad: \quad u_{c_{y}}(\cdot)\left[(1-\tau) w_{y}\right]-u_{1-l_{y}}(\cdot)=0, \\
S_{y} \quad: \quad-u_{c_{y}}(\cdot)+\pi(\cdot) V_{S_{y}}(\cdot)=0, \\
\quad \Leftrightarrow \quad-u_{c_{y}}(\cdot)+R v_{c_{o}}(\cdot)=0 \\
e_{y}^{p} \quad: \quad-u_{c_{y}}(\cdot)+\left[\frac{\partial \pi(\cdot)}{\partial e_{y}} V(\cdot)+\pi(\cdot) V_{e_{y}}(\cdot)\right] \frac{\partial e_{y}}{\partial e_{y}^{p}}=0, \\
\quad \Leftrightarrow \quad-u_{c_{y}}(\cdot)+\pi\left\{v_{h_{o}}(\cdot)+v_{c_{o}}(\cdot)(1-\tau) \frac{\partial w_{o}}{\partial h_{o}} l_{o}\right\} \frac{\partial h_{o}}{\partial e_{y}} \frac{\partial e_{y}}{\partial e_{y}^{p}}+\frac{\partial \pi(\cdot)}{\partial e_{y}} V(\cdot) \frac{\partial e_{y}}{\partial e_{y}^{p}}=0 .
\end{aligned}
$$

Since $\frac{\partial e_{y}}{\partial e_{y}^{p}}=1$ (public and private health investments are perfect substitutes), the first order condition for private health investment reduces to

$$
u_{c_{y}}(\cdot)=\pi\left\{v_{h_{o}}(\cdot)+v_{c_{o}}(\cdot)(1-\tau) \frac{\partial w_{o}}{\partial h_{o}} l_{o}\right\} \frac{\partial h_{o}}{\partial e_{y}}+\frac{\partial \pi(\cdot)}{\partial e_{y}} V(\cdot)
$$

The second order conditions for this problem are straightforward but tedious to write; henceforth, we assume they hold.

The interpretation of the first order optimality conditions for labor supply and saving is standard. The one for private health investment is as follows. An unit of the good invested in one's health reduces current felicity because it is no longer available for current consumption. On the plus side, such an investment has future benefits in the form of reduced mortality $\left(\frac{\partial \pi(\cdot)}{\partial e_{y}}\right)$ and 
reduced morbidity $\left(V_{e_{y}}(\cdot)\right)$; not to mention, the change in total health investment brought about by the unit increase in private investment, $\left(\frac{\partial e_{y}}{\partial e_{y}^{p}}\right)$.

An equilibrium with coexistence of private and public health investment is defined a 3-tuple $\left(c_{y}, c_{o}, e_{y}^{p}\right) \in \Re_{++}^{3}$ with $l_{y} \in(0,1), l_{o} \in(0,1)$ that solves $(4),(5),(7),(3)$, satisfies the relevant second-order conditions and the public sector budget constraint (2). An equilibrium with only private health investment is defined as above except it has $\tau=e_{y}^{g}=0$. An equilibrium with only public health investment is defined as above except it has $e_{y}^{p}=0$ and $e_{y}^{g}>0$. We assume all such equilibria exists.

\section{A Rationale For PUblic INVESTMENT In HeALth?}

We wish to know whether there is a welfare rationale for a policymaker to make publicly-funded investments in health (as a top-up on existing private investments). In our setting, the prima facie presumption has to be in the negative; after all, public and private health investments are assumed to be perfect substitutes, and the former is financed via a distortionary tax.

Consider a policy maker deciding on public health investments, $e_{y}^{g}$. The public sector budget constraint (2) implies that the tax rate needed to finance this investment is

$$
\tau=\frac{e_{y}^{g}}{w_{y} l_{y}+\pi(\cdot) w_{o}\left(h_{o}\right) l_{o}}
$$

We think of public health expenditures $\left(e_{y}^{g}\right)$ as the policy instrument, and therefore, under this interpretation, the tax rate is determined endogenously from (8).

A bit of detour on the tax-base effects of public health investment is in order. A change in public health investment has two direct effects on the tax base. It influences the survival probability thereby drawing in more to the tax base from the elderly. It also affects the health stock of agents and that affects their income. The combined effect on the income tax base, via its effect on the government's budget balance, affects the income tax rate, and thus the distortionary effects on labor supply, etc. To see this, use (8) to derive

$$
\frac{\partial \tau(\cdot)}{\partial e_{y}^{g}}=\frac{1}{w_{y} l_{y}+\pi(\cdot) w_{o}\left(h_{o}\right) l_{o}}-e_{y}^{g} \frac{w_{y} \frac{\partial l_{y}}{\partial \tau} \frac{\partial \tau(\cdot)}{\partial e_{y}^{g}}+\pi(\cdot) w_{o}\left(h_{o}\right) \frac{\partial l_{o}}{\partial \tau} \frac{\partial \tau(\cdot)}{\partial e_{y}^{g}}+\pi(\cdot) l_{o} \frac{\partial w_{o}\left(h_{o}\right)}{\partial e_{y}^{g}}+w_{o}\left(h_{o}\right) l_{o} \frac{\partial \pi(\cdot)}{\partial e_{y}} \frac{\partial e_{y}}{\partial e_{y}^{g}}}{\left[w_{y} l_{y}+\pi(\cdot) w_{o}\left(h_{o}\right) l_{o}\right]^{2}},
$$

which, using (8) and upon re-organization and simplification, yields the following expression for the tax elasticity:

$$
\frac{\partial \tau(\cdot)}{\partial e_{y}^{g}} \frac{e_{y}^{g}}{\tau}=1-[\underbrace{w_{y} \tau(\cdot) \frac{\partial l_{y}}{\partial \tau} \frac{\partial \tau(\cdot)}{\partial e_{y}^{g}}}_{\text {young labor distortion }}+\underbrace{\pi(\cdot) w_{o} \tau(\cdot) \frac{\partial l_{o}}{\partial \tau} \frac{\partial \tau(\cdot)}{\partial e_{y}^{g}}}_{\text {old labor distortion }}+\underbrace{\pi(\cdot) \tau(\cdot) l_{o} \frac{\partial w_{o}\left(h_{o}\right)}{\partial e_{y}^{g}}}_{\text {wage effect }}+\underbrace{w_{o} l_{o} \tau(\cdot) \frac{\partial \pi(\cdot)}{\partial e_{y}} \frac{\partial e_{y}}{\partial e_{y}^{g}}}_{\text {mortality effect }}]
$$


Even when labor supply is exogenous, that is the indirect labor distortion terms in (9) drop out, two direct effects remain: a) more public investment in health reduces morbidity and hence, the wages of the old increase (an important element of the tax base), b) more people survive to old age, further helping the tax base.

The policymaker is utilitarian, caring equally about the lifetime welfare of every two period-lived generation. ${ }^{12}$ The problem of the policy maker is described as

$$
\max _{e_{y}^{g}} \Upsilon \equiv u\left([1-\tau(\cdot)] w_{y} l_{y}-S_{y}-e_{y}^{p}, 1-l_{y}\right)+\pi\left(e_{y}\right) V\left(e_{y}, S_{y}, \tau, \tilde{R}\right)
$$

where the equilibrium value of $l_{y}$ is determined by (4), $S_{y}$ by (5), $e_{y}^{p}$ by (6), the tax rate $\tau$ follows from the budget constraint (8), and it is recognized that $\tilde{R}=R / \pi\left(e_{y}\right)$. Private agents make their choices taking the government's involvement as given; in turn, the latter makes its choices taking the private responses into account. The marginal welfare effect of a change in public health investment is captured by:

$$
\begin{aligned}
& \frac{\partial \Upsilon}{\partial e_{y}^{g}}=-u_{c_{y}}(\cdot) w_{y} l_{y} \frac{\partial \tau(\cdot)}{\partial e_{y}^{g}}+\left[u_{c_{y}}(\cdot)[1-\tau(\cdot)] w_{y}-u_{1-l_{y}}(\cdot)\right] \frac{\partial l_{y}}{\partial e_{y}^{g}} \\
& +\left[-u_{c_{y}}(\cdot)+R V_{S_{y}}(\cdot)\right] \frac{\partial S_{y}}{\partial e_{y}^{g}} \\
& +\left[-u_{c_{y}}(\cdot)+\frac{\partial \pi(\cdot)}{\partial e_{y}} V(\cdot)+\pi V_{e}(\cdot)-\pi V_{\tilde{R}}(\cdot) \frac{R}{\pi^{2}} \frac{\partial \pi(\cdot)}{\partial e_{y}}\right] \frac{\partial e_{y}^{p}}{\partial e_{y}^{g}} \\
& +\pi V_{\tau}(\cdot) \frac{\partial \tau(\cdot)}{\partial e_{y}^{g}}-V_{\tilde{R}}(\cdot) \frac{R \pi}{\pi^{2}} \frac{\partial \pi(\cdot)}{\partial e_{y}}+\frac{\partial \pi(\cdot)}{\partial e_{y}} V(\cdot)+\pi V_{e_{y}}(\cdot) .
\end{aligned}
$$

By the envelope theorem, any indirect effects on the optimal private choices of young labor supply and savings are washed out. Since private agents do not internalize the effect of their own health investment on the survival probability and on the overall budget, second order effects of $e_{y}^{g}$ on the optimal $e_{y}^{p}$ are not eliminated. Overall, changes in public health affect welfare through its direct effect on the budget and the survival probability, and indirectly, via its effect on the annuity interest rate and on private health investment (the crowding-out part). Using the first order conditions (4), $(5)$, and (6), we get

$$
\begin{aligned}
\frac{\partial \Upsilon}{\partial e_{y}^{g}} & =\underbrace{\left[-u_{c_{y}}(\cdot) w_{y} l_{y}+\pi V_{\tau}(\cdot)\right] \frac{\partial \tau(\cdot)}{\partial e_{y}^{g}}}_{\text {budget effect }}+\underbrace{\frac{\partial \pi(\cdot)}{\partial e_{y}} V(\cdot)+\pi V_{e_{y}}(\cdot)}_{\text {mortality effect }}-\underbrace{V_{\tilde{R}}(\cdot) \tilde{R} \frac{\partial \pi(\cdot)}{\partial e_{y}}}_{\text {interest rate effect crowding-out }} \underbrace{\left[1+\frac{\partial e_{y}^{p}}{\partial e_{y}^{g}}\right]} \\
& =\left[-u_{c_{y}}(\cdot) w_{y} l_{y}+\pi V_{\tau}(\cdot)\right] \frac{\partial \tau(\cdot)}{\partial e_{y}^{g}}+u_{c_{y}}(\cdot)-V_{\tilde{R}}(\cdot) \frac{R}{\pi} \frac{\partial \pi(\cdot)}{\partial e_{y}}\left[1+\frac{\partial e_{y}^{p}}{\partial e_{y}^{g}}\right],
\end{aligned}
$$

\footnotetext{
${ }^{12}$ See Section 4 for a discussion of the utilitarian yardstick and its implications for implementation of a public health policy.
} 
where in the last step, we use (6), i.e., $-u_{c_{y}}(\cdot)+\frac{\partial \pi(\cdot)}{\partial e_{y}} V(\cdot)+\pi V_{e_{y}}(\cdot)=0$. Using (5) and $V_{\tau}=$ $-v_{c_{o}}(\cdot) w_{o} l_{o}$, we can rewrite $(10)$ as

$$
\frac{\partial \Upsilon}{\partial e_{y}^{g}}=R v_{c_{o}}(\cdot)\{\underbrace{\left(1-\left[w_{y} l_{y}+\frac{\pi w_{o} l_{o}}{R}\right] \frac{\partial \tau(\cdot)}{\partial e_{y}^{g}}\right)}-S_{y} \frac{1}{\pi} \frac{\partial \pi(\cdot)}{\partial e_{y}}\left[1+\frac{\partial e_{y}^{p}}{\partial e_{y}^{g}}\right]\} .
$$

A unit of funds invested in public health changes the tax revenue (both current and future); the net benefit from this intergenerational transfer is captured by the underscored term. The second term on the right hand side of (11) arises because public health spending changes the mortality risk; whether it is raised or lowered depends on whether total health (private plus public) goes up or down and whether the concomitant changes in the annuity rate helps or hurts depends on whether the agent is a saver or a borrower. Overall, it is difficult to make an overall claim about the desirability of expanding public health.

We now turn to a narrower question: is there a welfare case for introducing public health in an initial equilibrium with positive private health investment? As shown in Appendix A, using (11), the marginal effect on steady state welfare of introducing a small increase in public health investment is given by

$$
\left.\frac{\partial \Upsilon}{\partial e_{y}^{g}}\right|_{e_{y}^{g}=0}=\left[(R-1) \frac{\pi(\cdot) w_{o}\left(h_{o}\right) l_{o}}{\left[w_{y} l_{y}+\pi(\cdot) w_{o}\left(h_{o}\right) l_{o}\right]}-S_{y} \tilde{R} \frac{\partial \pi(\cdot)}{\partial e_{y}}\left(1+\left.\frac{\partial e_{y}^{p}}{\partial e_{y}^{g}}\right|_{e_{y}^{g}=0}\right)\right] v_{c_{o}}(\cdot) .
$$

3.1. Exogenous mortality risk. We begin by considering a special, clean case in which health investments affect old-age morbidity but not the survival probability, i.e., $\frac{\partial \pi(\cdot)}{\partial e_{y}}=0$. This means, the second term in (12) drops out.

Proposition 1. If $R>1$ and $\frac{\partial \pi(\cdot)}{\partial e_{y}}=0$, then $\left.\frac{\partial \Upsilon}{\partial e_{y}^{g}}\right|_{e_{y}^{g}=0}>0$.

As is clear from (12), a small increase in public health investment raises welfare at the margin if the economy is dynamically efficient. More loosely, introducing public intervention in health may be justified if the economy is dynamically efficient and mortality risk is exogenous. Note this holds under the very general formulation of health and utility from both consumption and leisure. Also, the net asset position of the young does not matter.

It bears emphasis that this result relies crucially on the fact that the old work and are taxed. To see this, suppose the old work but are not taxed. Then the government's budget constraint becomes $e_{y}^{g}=\tau w_{y} l_{y}$, and hence $\frac{\partial \tau(\cdot)}{\partial e_{y}^{g}}=1 / w_{y} l_{y}$, and $V_{\tau}(\cdot)=0$. From (10), it follows that

$$
\frac{\partial \Upsilon}{\partial e_{y}^{g}}=\left[-u_{c_{y}}(\cdot) w_{y} l_{y}+\pi(\cdot) V_{\tau}(\cdot)\right] \frac{\partial \tau(\cdot)}{\partial e_{y}^{g}}+u_{c_{y}}(\cdot)=\left[-u_{c_{y}}(\cdot) w_{y} l_{y}\right] \frac{1}{w_{y} l_{y}}+u_{c_{y}}(\cdot)=0 .
$$

In other words, for Proposition 1 to hold, the intergenerational transfer mechanism has to be active. 
The intuition for this result is as follows. The private expected opportunity cost to the young of "investing" in their own health is $\pi(\cdot) \tilde{R}=R>1$. Whereas, when the current old transfer (via taxes) some resources to the current young (in a reverse pay-as-you-go scheme) to top-up the latter's own health investment, the opportunity cost the old face is 1 (no population growth). Therefore, at the margin it is cheaper to finance investments in health via the intergenerational transfer scheme than via the market.

A bit of context for this result is in order. This result is a mirror of the classic Aaron-Samuelson result in pension economics. In that context, introducing a pay-as-you-go pension (funded by a lump-sum tax on the young) is welfare reducing if the economy is dynamically efficient. There, the argument is that private agents face a return on saving equal to $R$, which, in a dynamicallyefficient economy, dominates the return (unity) attainable via the public pension system (equal to the population growth rate). The Aaron-Samuelson result shows, that in a dynamically efficient economy, it is a bad idea to save via the pension system - the intergenerational transfer mechanism, going from the young to the old, hurts welfare. In Proposition 1, we show if saving via the intergenerational transfer mechanism is bad, borrowing via the same scheme has to be good. The scheme we have described, effectively borrows from people whose opportunity cost of funds is 1 (under a constant population) and transfers to those whose opportunity cost is $R>1$, and that, we have established, is a welfare-improving move.

3.2. Endogenous mortality risk: The Philipson-Becker effect. When health investments increase the survival probability $\left(\frac{\partial \pi(\cdot)}{\partial e_{y}}>0\right)$, the result is a lot more nuanced since "general equilibrium" effects enter the discussion. In particular, since the survival probability affects the return to annuities, the net asset position of the young starts to matter. Specifically, an increase in the survival probability reduces the market return on life annuities; this reduces the interest burden of borrowers (helping them) but reduces the interest income of savers (hurting them).

We repeat (12) here so as to facilitate interpreting its various terms:

$$
\left.\frac{\partial \Upsilon}{\partial e_{y}^{g}}\right|_{e_{y}^{g}=0}=[\underbrace{(R-1) \frac{\pi(\cdot) w_{o}\left(h_{o}\right) l_{o}}{\left[w_{y} l_{y}+\pi(\cdot) w_{o}\left(h_{o}\right) l_{o}\right]}}_{\text {intergenerational transfer effect }}-\underbrace{S_{y} \tilde{R} \frac{\partial \pi(\cdot)}{\partial e_{y}}\left(1+\left.\frac{\partial e_{y}^{p}}{\partial e_{y}^{g}}\right|_{e_{y}^{g}=0}\right)}_{\text {Philipson-Becker effect }}] v_{c_{o}}(\cdot) .
$$

The Philipson-Becker effect, the second term in parenthesis on the r.h.s. of (13), arises if private individuals do not internalize the effect of their own health investments (via the survival probability) on the market return on life annuities. This causes $e_{y}^{p}$ to deviate from socially optimal levels, something the government can attempt to correct via $e_{y}^{g}$. Because changes in $e_{y}^{g}$ elicit changes in 
$e_{y}^{p}$ - crowding out - the extent of this correction matters; after all, the opportunity cost attached to $e_{y}^{g}$ and $e_{y}^{p}$ are very different, 1 versus $R$.

Specifically, we need to know, when $e_{y}^{g}$ increases, does $e_{y}=e_{y}^{p}+e_{y}^{g}$ fall or rise or stay unchanged? It follows from (13) that if the crowding out is exactly $100 \%$, i.e., $e_{y}$ (and hence, $\pi$ ) is unchanged, then again we are back to the result in Proposition 1. The next lemma argues the crowding out is, generically, never an exact $100 \%$.

Lemma 1. $\left.\frac{\partial e_{y}^{p}}{\partial e_{y}^{g}}\right|_{e_{y}^{g}=0} \neq-1$ holds generically.

More generally, we need to know if the crowding out is more or less than complete (i.e., does $e_{y}$ rise or fall when $e_{y}^{g}$ rises)? It turns out that an answer to this question, at the level of generality we are operating under, is unattainable. ${ }^{13}$ Crowding out is less than complete if

$$
\left.\frac{\partial e_{y}}{\partial e_{y}^{g}}\right|_{e_{y}^{g}=0}=1+\left.\frac{\partial e_{y}^{p}}{\partial e_{y}^{g}}\right|_{e_{y}^{g}=0}>0
$$

holds. This means, if (14) is true, total health investment $\left(e_{y}\right)$ increases (as does the survival probability), and the return on life annuities is reduced. We are now ready to state our main result.

Proposition 2. Suppose (14) holds. Then a) if $R>1$ and $S_{y}<0$, then $\left.\frac{\partial \Upsilon}{\partial e_{y}^{g}}\right|_{e_{y}^{g}=0}>0$ holds, and b) if $R>1$ and $S_{y}>0,\left.\frac{\partial \Upsilon}{\partial e_{y}^{g}}\right|_{e_{y}^{g}=0}$ is of ambiguous sign.

Under assumption (14), notably a sufficient condition, overall investment in health rises when the public component rises (even though the private part falls, due to crowding out). Young netborrowers $\left(S_{y}<0\right)$ clearly benefit if public health raises $\pi$, reduces the return on life annuities, and thereby eases their interest burden. In this case, from the societal standpoint, the aforediscussed Philipson-Becker effect implies that private individuals underinvest in their own health; they face a too low survival risk, and hence, too high an interest burden on their borrowing. From (12), it is clear that in this case, the intergenerational transfer effect (the part arising from $R$ exceeding unity) and the Philipson-Becker effect work in tandem to generate a role for public health. In fact, when the young are net borrowers, even if the intergenerational-transfer effect is shut down for some reason, the Philipson-Becker effect stays active and independently justifies public involvement in health. This means, even at the Golden Rule, the Philipson-Becker effect is enough to rationalize public health.

\footnotetext{
${ }^{13}$ Under some restrictive assumptions, including exogenous $l_{y}$ and $l_{o}$, one can show that $e_{y}$ rises when $e_{y}^{g}$ rises at the margin, locally near zero. A proof is available from the authors on request.
} 
When $S_{y}>0$, the situation is reversed. ${ }^{14}$ Public involvement in health reduces the return on life annuities and, ceteris paribus, this harms savers, and weakens the case for such policy action. In this case, the aforediscussed moral-hazard problem implies that private individuals overinvest in their own health, and any additional topping-up is harmful. A clear direction for welfare cannot be established for dynamically-efficient economies; in such cases, the intergenerational-transfer effect and the Philipson-Becker effect work at cross purposes.

3.3. Numerical experiments. More generally, it would be useful to know more about the desirability of introducing or expanding public health in settings where assumption (14) is violated or $S_{y}>0$. To make further progress, we resort to conducting several numerical experiments. ${ }^{15}$ To that end, assume (1) takes the commonly-used CRRA form

$$
\frac{\left(c_{y}\right)^{1-\sigma}}{1-\sigma}+\frac{\left(1-l_{y}\right)^{1-\sigma}}{1-\sigma}+\pi\left[\frac{\left(c_{o}\right)^{1-\sigma}}{1-\sigma}+\frac{\left(1-l_{o}\right)^{1-\sigma}}{1-\sigma}\right]
$$

where $\sigma<1$ is assumed to ensure $v()>$.0 . Also $\pi\left(e_{y}\right) \equiv \frac{e_{y}}{1+e_{y}} \in(0,1)$ and $w_{y}=w_{o} \equiv w$. It is easily verified that the relevant first order conditions are given by

$$
\begin{gathered}
l_{y}:\left((1-\tau) w l_{y}-S_{y}-e_{y}^{p}\right)^{-\sigma}((1-\tau) w)-\left(1-l_{y}\right)^{-\sigma}=0 \\
l_{o}:\left[(1-\tau) w l_{o}+\tilde{R} S_{y}\right]^{-\sigma}(1-\tau) w-\left(1-l_{o}\right)^{-\sigma} \\
S_{y}:-\left((1-\tau) w l_{y}-S_{y}-e_{y}^{p}\right)^{-\sigma}+\frac{\pi(\cdot) \tilde{R}}{((1-\tau) w)^{\frac{1}{\sigma}}+((1-\tau) w)}\left[\frac{((1-\tau) w)^{\frac{1}{\sigma}}}{\left((1-\tau) w l_{o}+\tilde{R} S_{y}\right)^{\sigma}}+\left(1-l_{o}\right)^{-\sigma}\right]=0,
\end{gathered}
$$

and

$$
e_{y}^{p}:-\left((1-\tau) w l_{y}-S_{y}-e_{y}^{p}\right)^{-\sigma}+\pi^{\prime}(\cdot)\left[\frac{\left((1-\tau) w l_{o}+\tilde{R} S_{y}\right)^{1-\sigma}}{1-\sigma}+\frac{\left(1-l_{o}\right)^{1-\sigma}}{1-\sigma}\right]=0
$$

Also, $\tau$ is computed from (8), and it is recognized that $\tilde{R}=R / \pi\left(e_{y}\right)$. The above equations are simultaneously solved to yield response functions, $l_{y}\left(e_{y}^{g}\right), l_{o}\left(e_{y}^{g}\right), S_{y}\left(e_{y}^{g}\right)$, and $e_{y}^{p}\left(e_{y}^{g}\right)$, converting $\Upsilon$ into a function of $e_{y}^{g}$.

Example 1. Suppose $\sigma=0.95, w=5$, and $R=1.1$. The panel below (Figure 1) illustrates various variables of interest as functions of $e_{y}^{g}$. As is evident from Figure 1, the welfare-maximizing level of $e_{y}^{g}$ is interior.

\footnotetext{
${ }^{14}$ Philipson and Becker (1998) split time into working and non-working (retirement) periods, and impose an assumption ensuring complete consumption smoothing. By implication, agents must have positive savings in the working period.

${ }^{15}$ Obviously, our intention here is not to conduct a full-blown calibration exercise, but simply to extend the scope of our analytical results.
} 

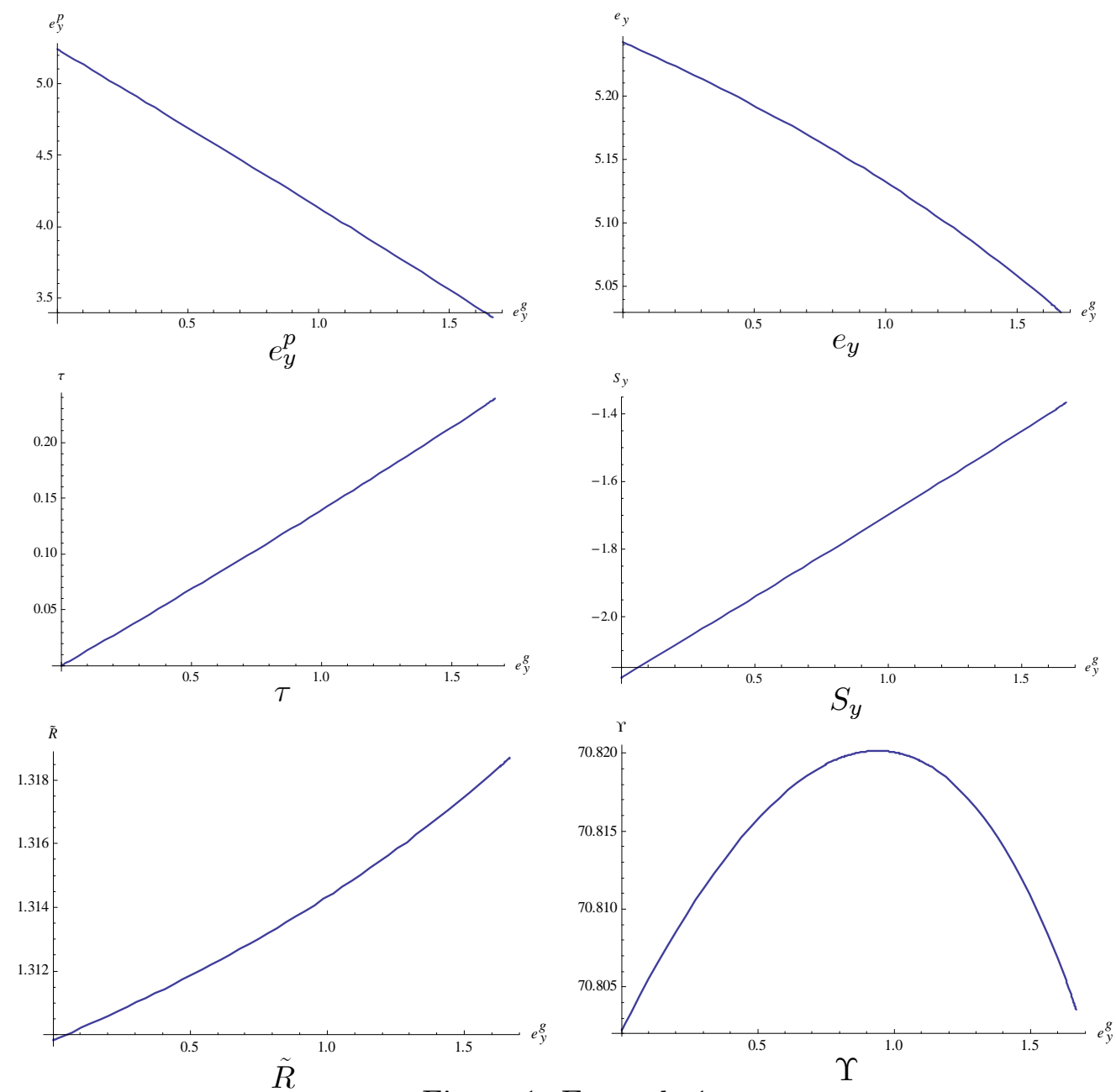

Figure 1: Example 1

Example 1 is a setting in which the young are net borrowers, and the economy is dynamically efficient. Here $e_{y}^{p}$ falls with an increase in $e_{y}^{g}$ so much so that total health spending, $e_{y}$, falls; i.e., crowding out is more than complete. This reduces $\pi$ and raises $\tilde{R}$. The higher public spending requires higher tax rates. Overall, social welfare is non-monotonic in $e_{y}^{g}$, and the optimal level of public health investments $e_{y}^{g}$ is positive.

Example 2. Suppose $\sigma=0.5, w=5$, and $R=1.25$. The panel below (Figure 2) illustrates various variables of interest as functions of $e_{y}^{g}$. As is evident from Figure 2, the welfare-maximizing level of $e_{y}^{g}$ is interior. 

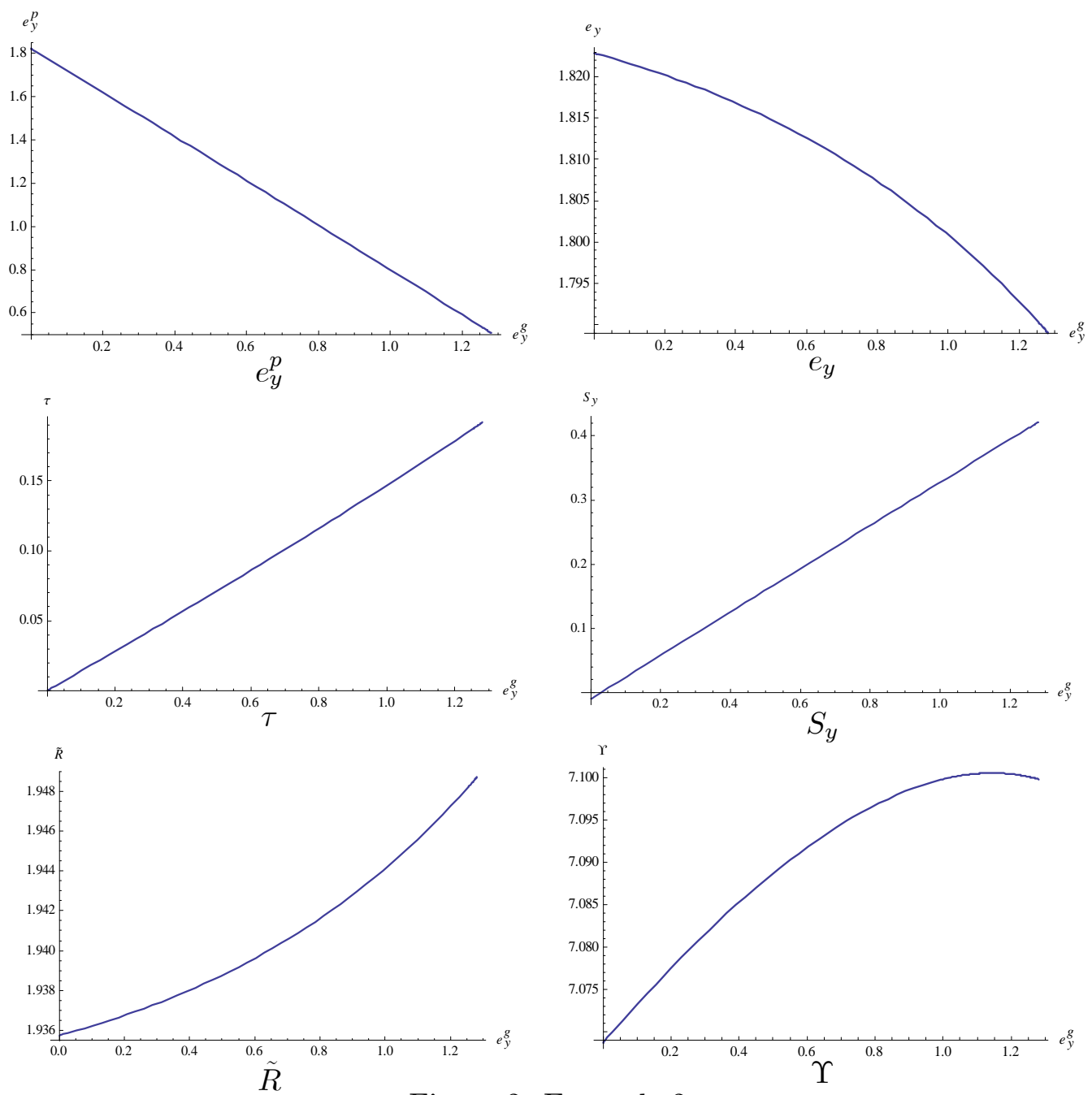

Figure 2: Example 2

Example 2 is a setting in which the young are net savers, and the economy is dynamically efficient. Here too $e_{y}^{p}$ falls with an increase in $e_{y}^{g}$ and total health spending falls reducing $\pi$ and raising $\tilde{R}$. The higher public spending requires higher tax rates. Overall, social welfare is again non-monotonic in $e_{y}^{g}$, and the optimal level of public health investments $e_{y}^{g}$ is positive.

\section{Concluding Remarks}

Public intervention in health is important and ubiquitous. Most often, it is rationalized by appealing to a host of market failures or distributional concerns. In this paper, we demonstrate that a role for government in preventive health activities may exist in their absence. We use a two-period model of endogenous mortality and morbidity inspired by Philipson and Becker (1998). Young agents invest a portion of labor income in their own health. Such investments reduce both mortality risk and old-age morbidity as well as improve general well-being. Public health activities are financed in a pay-as-you-go scheme where the government uses a distortionary tax on the labor income of 
the young and the old to finance spending on the young. Perfect substitutability of private and public investment in health is assumed, as is existence of competitive mortality-contingent claims "life-annuity" markets and dynamic efficiency. In such a setting, on the flip side of the classic Aaron-Samuelson result in pension economics, we prove that if survival risk is exogenous, then public intervention in health may be justified if the economy is dynamically efficient. ${ }^{16}$ If survival risk is endogenous, and private agents do not internalize the effect of their health investments on the survival probability, they may over or underinvest in health - the Philipson-Becker effect. We show if the young are net borrowers this effect strengthens the case for public health.

Our justification for using steady-state welfare as the yardstick is simple: if a policy fails to generate long-run welfare gains, it is unlikely to be ever adopted. However, despite our demonstration of the steady-state welfare gains, it may be questioned whether such an arrangement can ever be implemented in the short run (gotten off the ground). We have shown, when survival risk is exogenous, it is the intergenerational transfer mechanism that is essential to generating a welfare rationale for public health. ${ }^{17}$ In the language of Rangel (2003), the activity we consider is a forward-looking intergenerational good, one that is passed along an intergenerational link going forward from generation $t$ to generation $t+1$. For such a scheme to be implemented, there has to exist a first generation of old who contribute to it without having received any public health investment in their youth. Unless agents are altruistic, it appears our scheme cannot be implemented. However, as Rangel (2003) shows, implementation may be possible via a trigger strategy if the forward-looking intergenerational good is bundled with a backward-looking intergenerational good (passed on from generation $t+1$ to generation $t$ ) as happens with a pay-as-you-go pension. The intuition is that the support from generation $t$ for the forward-looking transfer to generation $t+1$ is ensured since their old-age pension is contingent on this support. Specifically, in our context, support for health (a forward-looking good) can presumably be ensured by bundling it with a backward-looking pension. ${ }^{18}$

\footnotetext{
${ }^{16}$ The main mechanism considered is not limited to health investment; indeed it applies (with some adjustments) to all expenditures made early in life that yield benefits later, such as education.

${ }^{17}$ Concerns about implementation do not arise when survival risk is endogenous. In that case, the Philipson-Becker, moral-hazard effect is operative. Even if the intergenerational transfer effect is shut down, public involvement in health may be justified for each and every generation if it ameliorates the moral hazard. The added nuance is that welfare effects depend on whether the young are net borrowers or savers.

${ }^{18}$ This intuition is readily transferred to the present setting with a minor reinterpretation of the model. Split up the period we have denoted old into middle-aged and retired and consider the following implementation of our health program. The middle-aged work and contribute to the financing of health which benefits the next generation. However, if the contribution to health is linked to a pension (received when retired) there is a linkage allowing trigger strategies to work. Current middle-aged will get a pension by the next generation only if they have contributed to the financing of health.
} 


\section{REFERENCES}

[1] Aaron, H., 1966, The Social Insurance Paradox, Canadian Journal of Economics, 32, 371-4.

[2] Augier, L., and A. Yaly, 2011. Economic Growth and Disease in the OLG Model, Working Paper, Université de Poitiers.

[3] Blanchard, O., and S. Fischer, 1989, Lectures on Macroeconomics, MIT Press.

[4] Blomgvist, A., and P.O. Johansson, 1997, Economic efficiency and mixed public/private insurance, Journal of Public Economics, 66, 505-516.

[5] Davidoff, T., J. R. Brown, and P. A. Diamond. 2005. "Annuities and Individual Welfare." American Economic Review, 95(5), 1573-1590.

[6] Davies, J.B., and P. Kuhn, 1992, Social Security, longevity and moral hazard, Journal of Public Economics, 49, 91-106.

[7] Diamond, P.A., 1965 National debt in a Neoclassical Growth Model, American Economic Review, 55(5), 1126-1150.

[8] Ehrlich, I., and H. Chuma. 1990. "A Model of the Demand for Longevity and the Value of Life Extension." Journal of Political Economy, 98(4): 761-82.

[9] Johansson, P.-O., 2000. Properties of actuarially fair and pay-as-you-go health insurance schemes for the elderly: an OLG approach, Journal of Health Economics, 19, 477-498.

[10] Gordon, R. L, E. L. Baker, W. L. Roper, and G. S. Omenn, 1996. "Prevention and the reforming U.S. Health Care System: Changing Roles and Responsibilities for Public Health", Annual Review of Public Health, 17, 489-509

[11] Grossman, M., 1972. On the Concept of Health Capital and the Demand for Health, Journal of Political Economy, 80(2), 223-255.

[12] Momota, A., K. Tabata, and K. Futagami, 2005. Infectious Disease and Preventive Behavior in an Overlapping Generations Model, Journal of Economic Dynamics and Control, 29, 16731700 .

[13] Liu, L., A.J. Rettenmaier, T.R. Saving, 2005. Longevity and Public Old-Age Pensions, Economic Inquiry, 43(2), 247-262.

[14] Philipson, T. J., and G. S. Becker, 1998. Old-age longevity and mortality-contingent claims, Journal of Political Economy, 106, 551-573.

[15] Rangel, A., 2003, Forward and Backward Intergenerational Goods: Why is Social Security Good for the Environment, American Economic Review, 93(3), 813-34.

[16] Schneider, M. T, and R. Winkler, 2010. Growth and Welfare under Endogenous Lifetime, Discussion paper 10-13, University of Bern.

[17] World Bank, 1993. Investing in Health: World Development Indicators. New York, NY: Oxford University Press.

[18] M. Yaari, 1965. Uncertain Lifetime, Life Insurance, and the Theory of the Consumer, Review of Economic Studies, 32(2), 137-50.

[19] Zhang, J., J. Zhang, and M.C.M. Leung, 2006. Health investment, saving, and public policy, Canadian Journal of Economics, 39, 68-93. 


\section{Appendix}

\section{A. Proof of Proposition 1}

We evaluate the marginal social value of increasing public health activities near $e_{y}^{g}=\tau=0$. First, note that

$$
\left.\frac{\partial \tau(\cdot)}{\partial e_{y}^{g}}\right|_{e_{y}^{g}=0}=\frac{1}{\left[w_{y} l_{y}+\pi(e) w_{o}\left(h_{o}\right) l_{o}\right]} .
$$

Using that $V_{\tau}(\cdot)=-v_{c_{o}}(\cdot) w_{o}\left(h_{o}\right) l_{o}$ we have

$$
\begin{aligned}
& {\left.\left[-u_{c_{y}}(\cdot) w_{y} l_{y}+\pi(\cdot) V_{\tau}(\cdot)\right] \frac{\partial \tau(\cdot)}{\partial e_{y}^{g}}\right|_{e_{y}^{g}=0}+u_{c_{y}}(\cdot)=-\frac{\left[u_{c_{y}}(\cdot) w_{y} l_{y}+\pi(\cdot) v_{c_{o}}(\cdot) w_{o}\left(h_{o}\right) l_{o}\right]}{\left[w_{y} l_{y}+\pi(\cdot) w_{o}\left(h_{o}\right) l_{o}\right]}+u_{c_{y}}(\cdot)} \\
& =v_{c_{o}}(\cdot)\left[(R-1) \frac{\pi(\cdot) w_{o}\left(h_{o}\right) l_{o}}{\left[w_{y} l_{y}+\pi(\cdot) w_{o}\left(h_{o}\right) l_{o}\right]}\right]
\end{aligned}
$$

where $-u_{c_{y}}(\cdot)+R v_{c_{o}}(\cdot)=0$ has been used. We thus have

$$
\left.\frac{\partial \Upsilon}{\partial e_{y}^{g}}\right|_{e_{y}^{g}=0}=(R-1) \frac{\pi(\cdot) w_{o}\left(h_{o}\right) l_{o} v_{c_{o}}(\cdot)}{\left[w_{y} l_{y}+\pi(\cdot) w_{o}\left(h_{o}\right) l_{o}\right]}-V_{\tilde{R}}(\cdot) \frac{R \pi(\cdot)}{(\pi(\cdot))^{2}} \pi_{e_{y}}(\cdot)\left(1+\frac{\partial e_{y}^{p}}{\partial e_{y}^{g}}\right)
$$

which is identical to (12). For a dynamically-efficient economy $(R>1)$, the first term is unambiguously positive, while the second term is ambiguously signed. If $\pi_{e_{y}}(\cdot)=0$, then clearly $\left.\frac{\partial \Upsilon}{\partial e_{y}^{g}}\right|_{e_{y}^{g}=0}$ $\forall R>1$.

\section{B. Proof of Lemma 1}

The following first shows that the equilibrium to the model can be stated in terms of total health expenditures $\left(e_{y}\right)$ and the tax rate $(\tau)$ and then turns to the crowding out issue. To recap, the model is summarized by the following first order conditions

$$
\begin{aligned}
& u_{c_{y}}\left(c_{y}, 1-l_{y}\right)-\pi \tilde{R} v_{c_{o}}\left(c_{o}, 1-l_{o}, h_{o}\left(e_{y}\right)\right)=0 \\
& -u_{1-l}\left(c_{y}, 1-l_{y}\right)+\pi \tilde{R} v_{c_{o}}\left(c_{o}, 1-l_{o}, h_{o}\left(e_{y}\right)\right)[1-\tau] w_{y}=0 \\
& \pi_{e}\left(e_{y}\right) v\left(c_{o}, 1-l_{o}, h_{o}\left(e_{y}\right)\right) \\
& +\pi\left(e_{y}\right)\left[v_{c}\left(c_{o}, 1-l_{o}, h_{o}\left(e_{y}\right)\right)\left([1-\tau] \frac{\partial w_{o}\left(h_{o}\right)}{\partial h} \frac{\partial h_{o}}{\partial e_{y}} l_{o}-\tilde{R}\right)+v_{h}\left(c_{o}, 1-l_{o}, h_{o}\left(e_{y}\right)\right) \frac{\partial h_{o}}{\partial e_{y}}\right]=0 \\
& v_{c_{o}}\left(c_{o}, 1-l_{o}, h_{o}\left(e_{y}\right)\right)[1-\tau] w_{o}\left(h_{o}\right)-v_{1-l_{o}}\left(c_{o}, 1-l_{o}, h_{o}\left(e_{y}\right)\right)=0
\end{aligned}
$$

and the budget constraint determining old age consumption

$$
\begin{aligned}
& c_{o}=[1-\tau] w_{o} l_{o}+\tilde{R} S_{y} \\
& =[1-\tau] w_{o} l_{o}+\tilde{R}\left[w_{y} l_{y}+\tau \pi(\cdot) w_{o}\left(h_{o}\right) l_{o}-c_{y}-e_{y}\right] .
\end{aligned}
$$

where

$$
e_{y}^{g}=\tau w_{y} l_{y}+\tau \pi(\cdot) w_{o}\left(h_{o}\right) l_{o}
$$


Using the expression for $c_{0}$ the equation system (16)-(19) can be rewritten in implicit form as

$$
\begin{aligned}
& f_{1}\left(\mathbf{z}^{\prime}, \tau\right)=0 \\
& f_{2}\left(\mathbf{z}^{\prime}, \tau\right)=0 \\
& f_{3}\left(\mathbf{z}^{\prime}, \tau\right)=0 \\
& f_{4}\left(\mathbf{z}^{\prime}, \tau\right)=0
\end{aligned}
$$

where $\mathbf{z}^{\prime} \equiv\left(z_{1}, z_{2}, z_{3}, z_{4}\right) \equiv\left(c_{y}, l_{y}, l_{o}, e_{y}\right)$. Note that all other exogenous variables than $\tau$ have been left out and that the level of public health is endogenously determined via the budget constraint. Total differentiation of the equation system (20)-(23) yields $\mathbf{A} d \mathbf{z}=-\mathbf{B} d \tau$ where

$$
\mathbf{A} \equiv\left[\begin{array}{llll}
a_{11} & a_{12} & a_{13} & a_{14} \\
a_{21} & a_{22} & a_{23} & a_{24} \\
a_{31} & a_{32} & a_{33} & a_{34} \\
a_{41} & a_{42} & a_{43} & a_{44}
\end{array}\right] ; \mathbf{B} \equiv\left[\begin{array}{c}
b_{1} \\
b_{2} \\
b_{3} \\
b_{4}
\end{array}\right]
$$

and $a_{i j} \equiv \frac{\partial f_{i}(z, \tau)}{\partial z_{j}} \neq 0$ for all $i, j$ and $b_{i} \equiv \frac{\partial f_{i}(z, \tau)}{\partial \tau} \neq 0$ for all $i$.

It follows from Cramer's rule that

$$
\frac{\partial e_{y}}{\partial \tau}=\frac{\left|\begin{array}{llll}
a_{11} & a_{12} & a_{13} & b_{1} \\
a_{21} & a_{22} & a_{23} & b_{2} \\
a_{31} & a_{32} & a_{33} & b_{3} \\
a_{41} & a_{42} & a_{43} & b_{4}
\end{array}\right|}{|\mathbf{A}|}=\frac{\left|\mathbf{A}_{1}\right|}{|\mathbf{A}|} \neq 0
$$

Note that $|\mathbf{A}| \neq 0$ is implied by existence of equilibrium. $\frac{\partial e_{y}}{\partial \tau} \neq 0$ requires that $\mathbf{A}_{1}$ is non singular, $\left|\mathbf{A}_{1}\right| \neq 0$, which holds generically. This implies $\left.\frac{\partial e_{y}}{\partial e_{y}^{g}}\right|_{e_{y}^{g}=0} \neq 0$ and hence $\left.\frac{\partial e_{y}^{p}}{\partial e_{y}^{g}}\right|_{e_{y}^{g}=0} \neq-1$. 